\title{
ANÁLISIS LINGÜÍSTICO DE LA JURISPRUDENCIA Y LA TRANSTEXTUALIDAD*
}

\section{Linguistic analysis of jurisprudence and transtextuality}

\author{
Camilo Guzmán Gómez** \\ Rodrigo González Quintero*** \\ Andrés Sarmiento Lamus****
}

Recepción: 13 de julio de 2019. Aceptación: 22 de junio de 2020

DOI: http://dx.doi.org/10.21017/Rev.Repub.2020.v29.a88

\section{RESUMEN}

El presente artículo de investigación busca demostrar la posibilidad epistemológica de aplicar métodos de análisis lingüístico al derecho y al estudio de la jurisprudencia, a través de la teoría de la transtextualidad de Gerard Genette. Por medio de este artículo se expone una adaptación de esta teoría para aplicarla al derecho. Lo que se pretende es establecer, de manera metódica, las relaciones que un texto jurídico o un fallo puede tener con otros textos para así poder entender el alcance de estos textos. Se propone igualmente una nueva metodología de la transtextualidad jurídica para un análisis global de un concepto o un análisis concreto de un caso. Por último, se dan dos ejemplos concretos de aplicación de esta teoría a través de la noción de efecto útil y a través del análisis de la decisión Didier del Consejo de Estado Francés.

Palabras clave: lingüística, transtextualidad, metodología, jurisprudencia, derecho comparado.

* Artículo producto del proyecto de investigación titulado La crisis de la Justicia, Grupo de Investigación CREAR (Universidad Sergio Arboleda-Bogotá) y Grupo de Investigación Joaquín Aarón Manjarrés (Universidad Sergio Arboleda-Santa Marta).

* Profesor titular de la Universidad Sergio Arboleda, título de maestría en Derecho público, Université Montesquieu, Bordeaux 4, doctorado en curso en Derecho público en la Universidad París Saclay, investigador sénior (MinCiencias). Línea de investigación: Análisis crítico de las instituciones.

*** Profesor investigador de la Universidad Sergio Arboleda - Santa Marta. Doctor y magíster en Derecho, Washington University in St. Louis, Estados Unidos. Profesor de Maestría en Derecho Constitucional y Derechos Humanos, Universidad Panamericana México, investigador sénior MinCiencias.

**** Docente investigador de la Universidad Sergio Arboleda, título de maestría, doctorado en curso en la Universidad de Leiden, investigador sénior (MinCiencias). Línea de investigación: Análisis crítico de las instituciones. 


\begin{abstract}
This article aims to demostrate the possibility of applying methods of linguistic analysis to the law studies and to the study of the jurisprudence, especially across the Gerard Genette's theory, the transtextuality. By means of this article there is exposed an adjustment of this theory to apply it to Law. What is claimed is to establish, in a methodical way, the relations that a juridical text or a judgement can have with other texts to be able to understand the scope of these texts. One proposes equally a new methodology of the juridical transtextuality for a global analysis of a concept or a concrete analysis of a case. Finally, there are given two concrete examples of application of this theory across the notion of Effet utile and across the analysis of the decision Didier of the French Conseil d'Etat.
\end{abstract}

Key-words: linguistics, transtextuality, methodology, jurisprudence, comparative law.

\title{
1. INTRODUCCIÓN
}

El cambio de paradigma en las fuentes del derecho que conoce nuestro país ha vuelto indispensable el estudio sistemático de la jurisprudencia. Más allá del debate de saber si la jurisprudencia es fuente auxiliar o principal en nuestro sistema, el análisis de esta es, para todo operador jurídico, una necesidad. Los recientes desarrollos jurisprudenciales en este sentido evidencian el alcance del sistema de precedentes y su implementación perenne en Colombia. Tan es así, que la Corte Suprema de Justica ha considerado, en su sentencia del 9 de febrero de 2009-30751, que el hecho de no utilizar una jurisprudencia bien establecida por un juez puede llevar al prevaricato y, por lo tanto, a la cárcel a dicho juez (Moreno, 2009).

Igualmente, el Código de Procedimiento Administrativo y de lo Contencioso Administrativo de 2011 impone una obligación a la Administración de tener en cuenta las sentencias de unificación jurisprudencial del Consejo de Estado para la aplicación uniforme de las normas del ordenamiento jurídico. Este ejemplo demuestra de manera clara la importancia de la jurisprudencia como fuente, pero también la importancia de entenderla y de saberla utilizar. Adicionalmente, el Código establece, en su artículo 104, la extensión de aplicación de estas sentencias de unificación cuando un ciudadano haga la petición, siempre y cuando haya identidad en los supuestos fácticos y jurídicos.

Paralelamente a estos fenómenos y como consecuencia lógica de estos, el desarrollo de teorías jurídicas sobre el estudio de la jurisprudencia se ha acrecenta- 
do en nuestro país; es necesario hacer especial mención a los estudios de Diego López (2006). Sin embargo, estas teorías, a pesar de ser excelentes insumos metodológicos y muy necesarios para el estudio del derecho, son en algunos casos insuficientes para entender los textos jurídicos en sus contextos.

\section{PROBLEMA DE INVESTIGACIÓN}

Las metodologías actuales de análisis jurisprudencial permiten establecer un mapa o árbol de las diferentes sentencias de una sola jurisdicción sobre un tema específico, mas no establecer en todos los casos, en un verdadero estudio o mapa sobre las fuentes, referencias, influencias que condicionan el entendimiento de la jurisprudencia, así como su alcance. En una época de cambio de fuentes y de internacionalización y globalización del derecho, un análisis de la jurisprudencia como un sistema autopoiético y autorreferente (Teubner, 1993) en algunos casos puede parecer limitado.

Por lo tanto, si bien es cierto que esta metodología constituye un primer paso fundamental en el estudio de la jurisprudencia, no abarca todas las posibilidades de análisis de esta y está limitada esencialmente al análisis de la jurisprudencia de la Corte Constitucional colombiana, por lo que el análisis de la jurisprudencia de otras jurisdicciones se dificulta con esta metodología.

\section{HIPÓTESIS DE TRABAJO}

Para resolver este problema epistemológico, es indispensable encontrar otras metodologías complementarias para la compresión global del derecho. Como premisa es indispensable partir del siguiente postulado: el derecho es lengua. Es decir, toda expresión del derecho necesita una formalización, y esta formalización se hace necesariamente a través de la lengua y de un texto. Aún el derecho no escrito, verbigracia la costumbre o los principios generales del derecho, para ser derecho, para ser normativo, necesita una formalización lingüística o textual. La noción de texto debe entenderse en un sentido amplio, es decir, puede cubrir varias realidades, fuentes o códigos. Se debe entender el texto como «dispositivo de información y comunicación que usa o actualiza reglas de códigos para hacer una propuesta de significado». (Naranjo P., González H., \& Rodríguez M., 2016, pág. 182). La práctica del sistema legal consiste en una representación textual de la norma que es aplicada y el evento social al cual se aplica la norma (Liste, 2010).

Así las cosas, la interpretación jurídica de los textos jurídicos es un trabajo complejo que se asemeja algunas veces a un rompecabezas compuesto de 
diferentes piezas y que requiere que el intérprete busque información por fuera del texto (Aarnio, 1987, pág. 112).

Por consiguiente, la implementación de técnicas de análisis lingüístico aplicadas al derecho permite una real comprensión del sentido, el alcance y el contenido de las diferentes expresiones del derecho: leyes, constitución, actos administrativos, jurisprudencia, costumbre. Se puede considerar que el estudio del derecho a través de la lingüística permite sobrepasar el estudio de los contenedores, es decir, las reglas para analizar concretamente los contenidos, las normas (De Béchillon, 1997) o las disposiciones jurídicas y las normas jurídicas (Corte Constitucional sentencia C-312/17, 2017).

La intertextualidad es una teoría o técnica de la lingüística contemporánea que fue desarrollada por la escuela francesa de la semiología partiendo de los estudios de Julia Kristeva, quien fue la creadora del término intertextualidad. Desde entonces, muchos autores han retomado este término y han creado diferentes teorías y definiciones; sin embargo, es Gérard Genette (2000) quien le dio su verdadero alcance práctico y sistemático, alejándose de las aproximaciones posestructurales (Allen, 2006, pág. 101).

Para Genette (2000), la intertextualidad o, mejor dicho, la transtextualidad, como él prefiere llamarla, corresponde a todo lo que "pone en relación, de manera manifiesta o secreta» ${ }^{1}$ un texto con otro (pág. 7). De este modo se le da una mayor perspectiva al texto y permite cambiar la atención del estudio del autor del texto hacia las fuentes del mismo y su contexto social (Porter, 1986).

En otras palabras, corresponde al análisis desde un punto de vista lingüístico, de toda relación que un texto puede tener con otro, ya sea esta relación directa o indirecta. Esta teoría se propone estudiar los diferentes niveles y tipos de relación en función de una tipología compleja y completa propuesta por Genette, así como por otros autores. Esta teoría propia del estudio lingüístico y literario se puede utilizar para el estudio de la jurisprudencia y del derecho en general.

Se pueden encontrar unos pocos estudios que tratan el tema de la transtextualidad aplicada al derecho, por ejemplo el libro de F. Ost y Van de Kerchove (2002), y otros estudios en Estados Unidos y a nivel internacional.

1 Traducción del autor.

Un revisión de bases de datos internacionales como Sciencedirect muestra que para intertextualidad hay un resultado global de 767 referencias, con un gran crecimiento en los últimos años (solo para el 2012 ya hay 22 referencias), pero al poner intertextualidad y derecho solo aparecen alrededor de 260 referencias y ninguna con relación directa con análisis de jurisprudencia. 
Sin embargo, no hay ningún estudio sobre la aplicación de la teoría de Genette a la jurisprudencia ${ }^{2}$. Algunos textos se concentran en la labor del traductor (Ferran, 2005) o el análisis específico de textos, sobre la citas en materia jurídica, empero, ningún estudio lo ha aplicado concretamente a la jurisprudencia y ni ha creado una herramienta metodológica. Esta investigación pretende desarrollar una metodología novedosa con el fin de suplir esta falencia.

\section{ESTRATEGIA METODOLÓGICA}

La presente investigación está basada en la interdisciplinariedad (derecho y lingüística), estudio casuístico de jurisprudencia y de derecho comparado. Se compuso de varias etapas diferentes. La primera encaminada a estudiar dogmáticamente la transtextualidad, principalmente los trabajos de Gérard Genette y en especial el libro Palimpsestos, la literatura en segundo grado. Una vez cumplida esta etapa, se realizó un trabajo de teorización y de adaptación de esta teoría para volverla aplicable al derecho. Finalmente, se realizaron aplicaciones concretas de esta teoría, primero desde una visión global y transtextual de un tema o concepto jurídico, y luego desde un análisis concreto de una sentencia.

\section{RESULTADOS}

El presente artículo se dividirá en dos partes, una teórica en la cual se expondrá la teoría y metodología de la transtextualidad (I) y una parte práctica en la cual se hará un estudio de la jurisprudencia sobre el efecto útil y el caso Didier del Consejo de Estado Francés (II).

\section{De la transtextualidad literaria a la transtextualidad jurídica}

\section{A. La transtextualidad}

La teoría desarrollada por Genette y los demás autores parte del principio de que todo texto (literario) es en realidad una suma de citas y referencias de textos anteriores, una especie de «collage» literario en el cual siempre es posible encontrar esas relaciones entre textos, así estas sean secretas o indirectas. Efectivamente como lector, siempre se tiene la impresión de que algún giro o frase ya fue leída anteriormente, lleva a algún recuerdo literario anterior, que esa idea ya había sido expuesta por otro autor, que la trama es la misma, entre muchas otras cosas. Es decir, por nuestra capacidad de asociación, permanentemente estamos asociando lo que leemos con lo que hemos leído o conocemos. Es más, muchas veces los autores mismos juegan con esto y el escrito se vuelve una red de citas, referencias o autorreferencias. Si no se utilizara esta capacidad 
de asociación sería imposible entender autores como Borges, Carpentier, Eco, Sollers, y obras tan esenciales como el Quijote de la Mancha que se encuentra atestado de referencias. De igual modo, muchas otras veces una relación intertextual puede ser el fruto del azar o puede ser inconsciente.

Para poder entender la lógica de la transtextualidad, es necesario partir de la idea de la existencia de una biblioteca universal o global, hoy podríamos decir, virtual, en la cual se encuentran todos los textos escritos en la historia de la humanidad. De esta biblioteca, tanto el escritor como el lector, pueden buscar las diferentes relaciones transtextuales que existen en el texto. Es igualmente necesario hacerse a la idea de que la relación existente entre los diferentes textos es una relación de red y no cronológica o jerárquica. Lo que equivale a decir que un texto cronológicamente posterior a otro puede darle una nueva lectura a un texto anterior y viceversa.

En otras palabras, el lector debe estar en una posición en la cual presente y pasado interactúan el uno sobre el otro, Sub specie aeternitatis (Rabau, 2002). Es más, un texto presente puede condicionar el futuro de la misma manera. La biblioteca universal se modifica entonces en permanencia.

Sin embargo, las relaciones entre textos son de diferente naturaleza; estas relaciones transtextuales pueden ser muy diversas. Por ello, Gérard Genette (2000) ideó cinco categorías diferentes plasmadas en su libro Palimpsestos y que servirán de base para el análisis transtextual del derecho y sobre todo de la jurisprudencia.

La primera categoría o tipo de transtextualidad es la intertextualidad. Para Genette (2000), «la intertextualidad corresponde a la relación de copresencia de dos o más textos (...) como la presencia efectiva de un texto en otro. Su forma más explícita y literal es la cita (con comillas, con o sin referencia precisa) (...) El plagio, que es una copia no declarada pero literal (...) La alusión, es decir, un enunciado cuya plena comprensión supone la percepción de su relación con otro enunciado al que remite necesariamente tal o cual de sus inflexiones, no perceptible de otro modo» ${ }^{2}$ (p. 8).

La segunda categoría descrita por Genette es la paratextualidad, que corresponde a la relación que tiene una obra con los diferentes componentes de su periferia textual, como por ejemplo los títulos, subtítulos, prefacio, postfacio, epígrafes, entre otros.

La tercera categoría expuesta por Genette es la metatextualidad, que es descrita como la relación crítica o de comentario de un texto con otro.

2 Traducción de los autores del artículo. 
El cuarto tipo de transcendencia textual es la hipertextualidad que reagrupa todo tipo de derivación o transformación que une un texto $B$ (hipertexto) a un texto anterior A (hipotexto) en el que se inserta de una manera que no es la del comentario.

Por último, encontramos la architextualidad, que es el género literario del texto.

Estas categorías corresponden a todos los tipos de relaciones transtextuales posibles, y a partir de estas construiremos una teoría de la transtextualidad jurídica.

\section{B. La transtextualidad como herramienta de la hermenéutica jurídica}

El análisis del Derecho así como las herramientas hermenéuticas son hoy en día un problema muy sensible por la multiplicación de las fuentes, sus horizontes diversos (ordenamiento jurídico internacional, jurisprudencia, etc.) y la complejidad creciente de estas. Sobre todo, cuando la teoría de la pirámide normativa es cada vez más cuestionada y hay nuevas teorías sobre la jerarquía de las fuentes y la creación de un sistema en red (Ost y Van de Kerchove, 2002) o de la Constitución Red (Bustos-Gisbert, 2005).

A nuestro parecer, la transtextualidad es un método idóneo para la investigación jurídica y el análisis cotidiano del derecho, pues esta teoría se adapta perfectamente a los nuevos desafíos del derecho. El análisis actual del derecho es un análisis sesgado, ya que en muchos casos se estudian los sistemas como sistemas cerrados; sin embargo, hoy en día estamos ante sistemas jurídicos abiertos. Por así decirlo, hay un cambio de paradigma en el estudio del Derecho, pues estamos pasando de un sistema autopoiético (Teubner, 1993), cerrado, de autorreferencialidad, a sistemas abiertos con fuentes, referencias, citas e influencias muy diversas.

La transtextualidad permite igualmente hacer un verdadero análisis científico, preciso y fundamentado de las relaciones entre textos jurídicos sin importar su origen o naturaleza. Se pasa entonces del simple análisis de las influencias o citas, para mostrar los reales niveles de relación entre diferentes textos jurídicos, ya sea jurisprudencia, costumbre, leyes, tratados, entre otros.

Por consiguiente, la transtextualidad puede integrarse dentro de las técnicas de la hermenéutica jurídica y como una herramienta metodológica para futuras investigaciones. Para ello, se realizó una esquematización y adaptación de esta teoría lingüística para volverla una teoría jurídica. Los principios básicos de la teoría literaria se pueden traspasar al estudio jurídico. 
La primera premisa que se puede adaptar de la teoría lingüística es la idea de una biblioteca jurídica global o universal. Los diferentes operadores jurídicos pueden utilizar cualquier texto, norma, principio, libro, doctrina, sin importar su origen ni el momento en que ese texto se escribió. El trabajo jurídico consiste la mayoría de las ocasiones en un trabajo de persuasión, la retórica jurídica consiste justamente en la persuasión. Para ello, los actores jurídicos utilizan diferentes técnicas argumentativas y una de ellas es la de citar o utilizar textos de referencia.

Por lo tanto, es muy común que los diferentes actores jurídicos lo hagan; las cortes utilizan doctrina y jurisprudencia extranjera cuando se enfrentan a un problema jurídico inédito, los abogados citan precedentes, el legislador se inspira de sistemas jurídicos foráneos.

La transtextualidad jurídica intenta entonces analizar esas técnicas argumentativas y retóricas para entender el trasfondo y las fuentes de las sentencias y textos jurídicos.

El segundo aspecto que podemos importar de la transtextualidad tiene que ver con la influencia que un texto jurídico tiene sobre el futuro, ya que puede definir o limitar su utilización transtextual posterior, tomando por ejemplo la modulación de las sentencias por parte de la Corte Constitucional, la reserva de interpretación y las sentencias de unificación.

En el sistema de precedentes al cual se dirige poco a poco el ordenamiento colombiano, la jurisprudencia se construye por estratos, lo que necesariamente lleva al condicionamiento de la jurisprudencia futura por la jurisprudencia actual y limita en cierto modo la labor del juez. Esta idea no es nueva en la teoría del Derecho, pues Ronald Dworkin (1994) considera que los jueces, que se asemejan en su trabajo a Hércules, escriben un libro o una novela global que tiene que ser lógica y tener continuidad y dar el mejor resultado posible para resolver los casos, creando así un derecho en cadena, en la cual cada sentencia es un eslabón más. Sin embargo, nuestra pretensión es ir más lejos y no limitarse al trabajo del juez sino tomar el derecho en su conjunto y bajo el entendido de que el derecho no es necesariamente cronológico.

La importancia de una sentencia se tiene en cuenta ya no desde un punto de vista cronológico sino por la importancia de los cambios que pueda operar del conjunto de la biblioteca jurídica. Todo texto determina retrospectivamente la interpretación de los textos precedentes y los textos precedentes, la creación de los textos futuros.

Tercero, podemos utilizar las categorías establecidas por Genette (2000) para adaptarlas a los tipos de relaciones entre textos jurídicos: 
La intertextualidad jurídica es la base misma del ejercicio de la construcción de textos jurídicos y se materializa por medio de la aplicación y la cita de normas, las normas de aplicación y de ejecución de otras normas, integración de normas internacionales en el ordenamiento interno (Bloque de Constitucionalidad), cita de precedentes jurisprudenciales, alusión a otros sistemas jurídicos, a principios generales del Derecho, Costumbre, entre otros.

La paratextualidad jurídica (periferia del texto) es importante para la interpretación jurídica, pues incluye trabajos preparatorios, exposición de motivos de normas, aclaraciones de voto, salvamento de voto de jueces, conclusiones de comisarios de gobierno, intervenciones del ministerio público, entre otros, que en muchos casos son fundamentales para entender un texto.

La metatextualidad jurídica (crítica o comentario) en materia jurídica se evidenciaría a través de comentarios de normas, comentarios de jurisprudencia y doctrina.

La hipertextualidad jurídica (derivación o transformación) es fundamental y corresponde a la transformación que conocen los textos jurídicos a través de otros textos o de su interpretación o reinterpretación. En esta categoría podemos encontrar, entre otras, normas total o parcialmente derogatorias de otras normas, normas modificatorias de normas anteriores, reservas a los tratados, declaraciones interpretativas a los tratados, sentencias modulativas, interpretativas, omisiones legislativas, cambio de interpretación de una norma o concepto, Sentencias de Unificación, y apartamiento y/o cambio de precedente jurisprudencial.

La única categoría que no vamos a utilizar es la de la architextualidad, que en este caso no es funcional, ya que en derecho el estilo literario que vamos a confrontar es el estilo jurídico.

A continuación, estableceremos un tablero sobre las relaciones transtextuales anteriormente descritas que tiene por objetivo principal simplificar la comprensión de la teoría de Genette (2000).

\begin{tabular}{|c|c|l|l|}
\hline Intertextualidad & Paratextualidad & Metatextualidad & Hipertextualidad \\
\hline Cita & Título, subtítulo & Comentario & Transformación \\
\hline Alusión & Prefacio, postfacio & Crítica & Imitación \\
\hline Plagio & Ilustraciones & & Sátira \\
\hline
\end{tabular}


$\mathrm{Si}$ adaptamos figuras de la transtextualidad literaria expuestas en el tablero anterior a la transtextualidad jurídica o del texto jurídico, el resultado sería el siguiente:

\begin{tabular}{|c|c|c|c|}
\hline Intertextualidad & Paratextualidad & Metatextualidad & Hipertextualidad \\
\hline $\begin{array}{l}\text { Cita de normas de } \\
\text { referencia; normas de } \\
\text { aplicación y de } \\
\text { ejecución de otras } \\
\text { normas; integración de } \\
\text { normas internacionales } \\
\text { en el ordenamiento } \\
\text { interno (Bloque de } \\
\text { Constitucionalidad). }\end{array}$ & Trabajos preparatorios & Comentarios de normas & $\begin{array}{l}\text { Normas total o parcialmente } \\
\text { derogatorias de otras normas. } \\
\text { Normas modificatorias de } \\
\text { normas anteriores. }\end{array}$ \\
\hline $\begin{array}{l}\text { Cita de precedentes } \\
\text { jurisprudenciales }\end{array}$ & $\begin{array}{c}\text { Exposición de motivos } \\
\text { de normas }\end{array}$ & $\begin{array}{l}\text { Comentarios de } \\
\text { jurisprudencia }\end{array}$ & $\begin{array}{c}\text { Reservas a los tratados; } \\
\text { declaraciones interpretativas a } \\
\text { los tratados. }\end{array}$ \\
\hline $\begin{array}{c}\text { Alusión a otros } \\
\text { sistemas jurídicos, a } \\
\text { principios generales del } \\
\text { Derecho, Costumbre. }\end{array}$ & $\begin{array}{l}\text { Aclaraciones de voto, } \\
\text { salvamento de voto de } \\
\text { jueces. Conclusiones } \\
\text { comisarios de gobierno, } \\
\text { intervenciones del } \\
\text { ministerio público. }\end{array}$ & Doctrina & $\begin{array}{c}\text { Sentencias modulativas, } \\
\text { interpretativas, de unificación, } \\
\text { omisiones legislativas, cambio } \\
\text { de interpretación de una norma } \\
\text { o concepto, apartamiento y/o } \\
\text { cambio de precedente } \\
\text { jurisprudencial. }\end{array}$ \\
\hline
\end{tabular}

A partir de este tablero consideramos que se pueden entender las diferentes relaciones transtextuales que puede haber en el derecho en general y en la jurisprudencia en particular y se puede crear la teoría de la aplicación de la transtextualidad al Derecho.

Esta aplicación puede ser general, para el análisis de un concepto o aplicada a una sentencia en particular.

\section{Aplicación concreta de la transtextualidad al derecho: el efecto útil y el debido proceso}

La transtextualidad jurídica tiene, desde nuestro punto de vista, dos tipos o maneras de aplicación posibles. La primera, que llamaremos análisis integral 
de la transtextualidad jurídica, consiste en tomar un concepto o una noción jurídica y ver su utilización por parte de la jurisprudencia. Es decir, se podría hacer un trabajo de semasiología transtextual jurídica ${ }^{3}$ en la cual se haría un estudio que, partiendo del concepto y de sus relaciones transtextuales, se propone la determinación del concepto.

La segunda aplicación, que llamaremos análisis concreto o casuístico de transtextualidad jurídica, consiste en tomar un texto concreto y ver las relaciones transtextuales que puede tener este texto. Es decir, en nuestro caso, tomar una sentencia y ver cuáles serían las diferentes formas de utilización de otros textos por esa sentencia.

Para dar herramientas suficientes para utilizar esta metodología que se propone, se expondrán ejemplos de la utilización de la transtextualidad jurídica en sus dos vertientes.

\section{A. Análisis global del concepto de efecto útil}

La noción de efecto útil es una herramienta de hermenéutica jurídica que consiste en interpretar una norma para darle sentido a los conceptos de la norma o para darle el mayor alcance posible. Esto viene del principio conocido en latín como ut res magis valeat quam pereat.

Este principio viene esencialmente del derecho internacional público para la interpretación de los tratados y fue utilizado por la Corte Internacional de Justicia en su fallo Corfu (1949).

Sin embargo, este principio toma real relevancia con la aplicación hecha por la Corte de Justicia de la Comunidad Europea (CJCE), quien le dio un mayor alcance al concepto esencialmente a partir del fallo Van Duyn (1974). Desde entonces, la jurisprudencia de la Corte ha desarrollado de manera muy importante este concepto, esencialmente para poder exigir la aplicación de las directivas europeas a los Estados miembros.

La Corte Interamericana de Derechos Humanos, por su parte, ha considerado que los jueces deben velar «porque el efecto útil de la Convención no se vea mermado o anulado por la aplicación de leyes contrarias a sus disposiciones, objeto y fin» (CIDH, 2006).

3 Se debe aclarar que esta expresión no existe como tal, pero se crea para una mejor comprensión de la transtextualidad jurídica. 
La Corte Constitucional colombiana ha utilizado este principio en incontables ocasiones y con múltiples fines y desde el inicio de su labor; tomaremos como ejemplo la sentencia T 001 (1992), en la cual la Corte estima que el principio implica que «entre dos posibles sentidos de un precepto, uno de los cuales produce consecuencias jurídicas y el otro a nada conduce, debe preferirse el primero». ${ }^{4}$

El Consejo de Estado, a su vez, ha utilizado el principio del efecto útil en el sentido de principio de interpretación de las normas jurídicas, al considerar que «entre dos posibles sentidos de un precepto, uno de los cuales produce efectos jurídicos y el otro a nada conduce, debe preferirse el primero» (Consejo de Estado, 2019a). Pero de igual modo, ha utilizado el principio en el sentido de garantizar el cumplimiento de las disposiciones de la Convención Americana de Derechos Humanos (Consejo de Estado, 2019b).

Por último, la Corte Suprema de Justicia en su sentencia 2001-06915-01 (2008) expone lo siguiente:

«frente a estipulaciones polisémicas, dicotómicas o patológicas "[e]l sentido en que una cláusula puede producir algún efecto, deberá preferirse a aquel en que no sea capaz de producir efecto alguno" (art. 1620 C.C.), privilegiándose la conservación del negocio jurídico, la utilidad respecto de la irrelevancia y la eficacia sobre la ineficacia del acto (effet utile, res magis valeat quam pereat)».

Podemos ver que el concepto de efecto útil tiene diferentes definiciones posibles y diferentes fuentes posibles. Si bien es cierto que todas tienen como ancestro común el principio general de interpretación, las variaciones son muchas.

Si quisiéramos establecer las relaciones transtextuales de estas diferentes posturas, daría muchos resultados. Básicamente hay dos tipos de relaciones transtextuales, la de intertextualidad y la de hipertextualidad:

1. El fallo Van Duyn tendría una relación intertextual y de hipertextualidad porque a la vez cita el principio general del derecho internacional que utiliza la Corte Internacional de Justicia, pero al mismo tiempo transforma el concepto y le da otro alcance.

2. El fallo de la Corte Interamericana tiene una relación intertextual con el principio general, a la Convención (art. 29), a precedentes anteriores propios.

4 La Corte ha utilizado este principio de muchas otras maneras, pero por las razones anteriormente especificadas, solo se utilizará esta sentencia. 
3. El fallo de la Corte Constitucional podríamos considerar que tiene una relación intertextual por medio de la alusión que hace del principio; hipertextual también, ya que durante la integración de este principio internacional le da en esta sentencia específica un alcance limitado o si se mira desde el punto de vista de la sentencia de la CJCE.

4. Los fallos del Consejo de Estado, por su parte, simplemente tendrían una relación intertextual con el principio general, con el precedente de la Corte Constitucional en el primer caso y con el precedente de la CIDH en el segundo; y se podría decir hipertextual con la sentencia de la CJCE, ya que la solución dada es diferente de lo propuesto por esta jurisdicción.

5. Por último, la sentencia de la Corte Suprema tiene una relación intertextual con el artículo 1620 del Código Civil, ya que lo cita textualmente, con el principio general y la locución latina. Sin embargo, tiene una relación hipertextual con la sentencia de la CJCE, de la Corte constitucional, de la CIDH y el Consejo de Estado.

Este ejercicio que se presenta de manera simplificada, por razones metodológicas, simplemente demuestra la cantidad de relaciones transtextuales que puede tener un concepto y permite elucidar al operador jurídico la noción, así como los argumentos que debe o puede utilizar en función de la jurisdicción ante la cual se encuentre.

De este análisis se puede concluir que el principio del efecto útil tiene dos vertientes diferentes; por un lado, es un criterio de interpretación de normas para darles el mayor o mejor alcance posible, y por el otro, es un principio que garantiza la aplicabilidad de normas internacionales en ordenamientos jurídicos internos. Estas dos definiciones del concepto quedan así claramente establecidas de modo que permiten esclarecer la noción.

\section{B. Análisis concreto del Caso Didier del Consejo de Estado Francés}

El fallo Didier (1999) del Consejo de Estado francés es un fallo emblemático y que ha sido considerado como uno de los grandes fallos de la jurisprudencia administrativa. Al hacer un análisis concreto de transtextualidad, todo en el caso importa, no solo su contenido sino también su periferia, su contexto.

El problema jurídico en el caso Didier era saber si se debía aplicar el artículo 6.1 de la Convención Europea de Derechos Humanos, que versa sobre el 
principio del debido proceso, y la jurisprudencia del Tribunal Europeo de Derechos humanos sobre la aplicación de este principio a las actuaciones donde la administración haga uso de su potestad sancionatoria. En otras palabras, se buscaba determinar si las sanciones de una superintendencia debían respetar el debido proceso.

Es menester, para la comprensión de la problemática general, entender que el Consejo de Estado francés siempre ha tenido una marcada resistencia a la aplicación directa de la Convención y a utilizar como fuente la jurisprudencia del Tribunal. Es más, los ciudadanos franceses debieron esperar hasta 1989, fallo Nicolo, para que el Consejo aceptara la aplicación del derecho internacional. Sin embargo, la jurisprudencia del Tribunal europeo considera, desde el caso Lecompte (1981), que el artículo 6 de la Convención se debe aplicar siempre que las sanciones de la administración tengan repercusiones civiles o penales.

Ante la perspectiva de ver su postura sancionada o criticada por el Tribunal Europeo, el Consejo de Estado debía encontrar una solución, sobre todo porque en los mismos casos la Corte de Casación Francesa sí aplicaba directamente el artículo 6 de la Convención Europea. El Consejo de Estado encontró una solución original que solo el análisis intertextual permite entender.

En efecto, en el fallo Didier, el Consejo de Estado se ve obligado a citar y hablar de la Convención Europea. Sin embargo, el análisis transtextual de la decisión demuestra que el Consejo no aplica la Convención, pese a citarla, sino que utiliza el principio general del derecho de imparcialidad para solucionar el conflicto y para tener una jurisprudencia propia, diferente de la europea y que puede moldear en función de sus necesidades.

Este fallo es interesante para nuestra teoría, pues cubre todas las relaciones transtextuales posibles y es un paradigma de la complejidad del derecho.

Primero, el fallo tiene una relación intertextual con la Convención, pues la cita directamente en varias ocasiones. Segundo, podemos ver una relación de paratextualidad, ya que la solución dada está basada en las conclusiones del Comisario de Gobierno, Seban, quien es el que nos indica que se utilizará el principio general del derecho y un viejo precedente Louis (1957). Tercero, en cuanto a la metatextualidad, durante el fallo, el Consejo de Estado y en las conclusiones se analiza y comenta el artículo 6 y su aplicabilidad al caso. Por último, existe una relación hipertextual con la jurisprudencia Louis (1957) y la jurisprudencia Lecompte (1981), pues la solución difiere de estas últimas para proponer una solución original. 
Si intentamos esquematizar el fallo Didier, tendríamos los siguientes resultados:

\begin{tabular}{|c|c|c|c|}
\hline Intertextualidad & Paratextualidad & Metatextualidad & Hipertextualidad \\
\hline $\begin{array}{c}\text { Cita de la Convención } \\
\text { Europea de Derechos } \\
\text { Humanos y alusión al } \\
\text { Principio general del } \\
\text { derecho. }\end{array}$ & $\begin{array}{c}\text { Conclusiones del } \\
\text { Comisario de Gobierno } \\
\text { A. Seban. }\end{array}$ & $\begin{array}{l}\text { Comentarios de la } \\
\text { Convención y del } \\
\text { principio general del } \\
\text { derecho de } \\
\text { imparcialidad. }\end{array}$ & $\begin{array}{c}\text { Transformación del } \\
\text { precedente Louis (1957). }\end{array}$ \\
\hline
\end{tabular}

Sin un análisis transtextual del fallo Didier, no sería posible entender el alcance de este; sin leer o conocer las conclusiones del Comisario de Gobierno (paratextualidad), no se puede entender la sentencia, y al mismo tiempo, sin leer el fallo Louis (hipertextualidad), no se puede entender la novedad de la postura del Consejo de Estado. Posteriormente, el Tribunal Europeo consideró que la jurisprudencia Didier era válida y suficiente para cumplir con las obligaciones que impone el artículo 6-1 de la Convención Europea de Derechos humanos. El análisis transtextual permite concluir que es falso decir que el Consejo de Estado aplica directamente la Convención (como lo dijo parte de la doctrina), así como lo es decir que aplica el fallo Louis, pues en realidad se trata de una concepción nueva ${ }^{5}$ del principio de imparcialidad y su aplicación a las actuaciones de la administración.

Por lo tanto, lo primordial en la comprensión del texto, del fallo, no es la norma citada y que parece ser el fundamento de este (intertextualidad), sino la relación paratextual y posteriormente la relación hipertextual y metatextual.

\section{CONCLUSIONES}

A través del estudio de la transtextualidad y de la transtextualidad jurídica aplicada a casos específicos queda claro, a nuestro parecer, que el estudio del derecho debe tener en cuenta la lingüística como herramienta interpretativa y metodológica y que utilizar esta herramienta puede llevar a un análisis más amplio y en contexto de la realidad jurídica. La transtextualidad se impone entonces como un método de fácil utilización por todo operador jurídico, rico

5 Nueva para el Consejo de Estado Francés, aunque muy similar a la del Tribunal Europeo, el Consejo Constitucional francés y la Corte de Casación, pues los efectos son similares. 
en los resultados que puede arrojar y práctico, pues otorga herramientas para el análisis de textos jurídicos en diferentes niveles.

\section{BIBLIOGRAFÍA}

\section{Libros y artículos}

Aarnio, A. (1987). Sobre la ambigüedad semántica en la interpretración jurídica Doxa(4), 109-177. doi:10.14198/DOXA1987.4.07

Allen, G. (2006). Intertextuality. London: Taylor \& Francis e-Library.

Bustos-Gisbert, R. (2005). La Constitución Red: un estudio sobre supraestatalidad y Constitución. Instituto Vasco de Administración Pública.

De Béchillon, D. (1997). Qu'est-ce qu'une règle de droit, Paris, Odile Jacob.

Dworkin, R. (1994). L'empire du droit, Paris, PUF.

Ferran, E. (2005). «La intertextualidad en el derecho y en la lingüística. La intertextualidad entre los documentos jurídicos de una cadena. Un análisis para la traducción», Meta : journal des traducteurs / Meta: Translators' Journal, vol. $50, n^{\circ} 4$.

Genette, G. (2000). Palimsestes, la littérature au second degré, Paris, Editions du Seuil.

Liste, P. (2010). The Politics of (Legal) Intertextuality. International Political Sociology 3, 319-322. DOI: 10.1111/j.1749-5687.2010.00107_6.x

López, D. (2006). El derecho de los jueces, Bogotá D. C., Legis.

Moreno, L. J. (2009). en Cuadernos de Derecho Público 1, Bogotá, Universidad Sergio Arboleda p. 5.

Naranjo P., S., González H., D. L., \& Rodríguez M., J. (julio-diciembre de 2016). El artículo científico: los límites de la interpretación de un género textual. Revista Republicana (21), 179-200. DOI: http:/ / dx.doi.org/10.21017/Rev.Repub.2016.v21.a17

Ost, F., y Van de Kerchove, M. (2002), De la pyramide au réseau ? Pour une théorie dialectique du droit, Bruselas, Publications des facultés universitaires Saint-Louis.

Porter, E. J.(1986). Intertextuality and Discourse Community. Rhetoric Review, 5(1). Taylor \& Francis, http:/ / www.jstor.org/stable/466015 
Rabau, S. (2002). L’intertextualité, Paris, GF Flammarion.

Teubner, G. (1993). Le droit, un sistème autopoiétique, PUF.

\section{Jurisprudencia}

Consejo de Estado francés, Ass, caso Didier (1999).

Consejo de Estado Francés, Ass, Caso Nicolo (1989).

Consejo de Estado Francés, Louis (1957).

Consejo de Estado, 11001-03-24-000-2009-00511-00 (2019a).

Consejo de Estado, 11001-03-25-000-2013-00943-00(2084-13) (2019b).

Corte Constitucional, sentencia T 001 (1992).

Corte Constitucional, sentencia C-312 (2017).

Corte Interamericana de Derechos Humanos, Caso Trabajadores Cesados del Congreso (2006).

Corte Internacional de Justicia, Corfu (1949).

Corte de Justicia de la Comunidad Europea, Van Dyun (1974).

Corte Suprema de Justicia, 2001-06915-01 (2008).

Tribunal Europeo de Derechos Humanos, Le Compte, Van Leuven et De Meyere C. Belgique (1981). 
